\title{
Tratamiento del Factor Tubo-Peritoneal con Microcirugía
}

\author{
Dr. Luis Ernesto Pérez
}

\section{INTRODUCCION}

Durante la última década la microcirugía tubárica reconstructiva se ha convertido en el procedimiento terapéutico más importante de la infertilidad de causa tubo-peritoneal (1).

La explosión tecnológica de la Biología Reproductiva ha producido tremendos avances en las técnicas microquirúrgicas de tal suerte que la microcirugía también ha sido empleada para estudiar la fisiología tubárica en animales de experimentación en el laboratorio $(2,3,4$, $5,6,7,8,9,10,11)$. Su importancia en la práctica clínica ha sido demostrada para la reversión de la ligadura de trompas en las grandes series de Population Reports (12) en donde se demuestra que por técnicas quirúrgicas convencionales desde 1940 a 1953 solo fue posible lograr embarazos en el $16 \%$ de 92 pacientes; con cirugía atraumática sin magnificación desde 1953 a 1976 se tuvo éxito en el $30 \%$ de 111 intervenciones; y con microcirugía se ha logrado desde 1971 a 1980 nacimientos de niños normales en el $56 \%$ de 560 pacientes operadas. La aplicación de la microciru-

Departamentos de Ginecologia y Obstetricia Hospital Militar Central

Clínica San Pedro Claver-ISS gía en el tratamiento de la endometriosis y de la obstrucción tubárica de causa infecciosa también ha duplicado y triplicado las tasas de éxitos que se obtenían por macrocirugía $(13,14)$.

El presente trabajo tiene por objeto presentar los resultados obtenidos en 72 pacientes infértiles por diversas patologías tuboperitoneales clasificadas según las normas internacionales y tratados con microcirugía.

\section{MATERIAL Y METODOS}

Se incluyen a setenta y dos pacientes con edades comprendidas entre 21 y 37 años a quienes habiendo consultado en el Hospital Militar Central de Bogotá o en la Clínica San Pedro Claver del ISS, se les diagnosticó infertilidad por factor tuboperitoneal. Una historia clínica y exámen físico completos, se realizó a toda pareja infértil y ante la sospecha de factor tuboperitoneal, la laparoscopia diagnóstica y la histerosalpingografía fueron los elementos de juicio para clasificar la patología tubo-peritoneal y las posibilidades de tratamiento microquirúrgico. A las candidatas a microcirugía se les explicó las posibilidades de un embarazo normal postratamiento en porcentajes que oscilaron entre el $10 \% \mathrm{y}$ el $70 \%$ que correspondían a los hidrosalpinx y la recanalización post-ligadura de trompas respectivamente, pasando por el 
resto de grupos patológicos. A las parejas que aceptaron la microcirugía, se les completó el estudio básico de la infertilidad según protocolo establecido para el efecto $y$ que incluye: espermograma, prueba post-coito y pruebas de ovulación (figs 1 y 2). La microcirugía se contraindicó en parejas con factores asociados tales como factor masculino, cervical y/o ovulatorio; sin embargo, muchas parejas con factores asociados solicitaron encarecidamente la microcirugía paralela al tratamiento de los demás problemas, pero ellas son objeto de otros estudios actualmente en progreso.

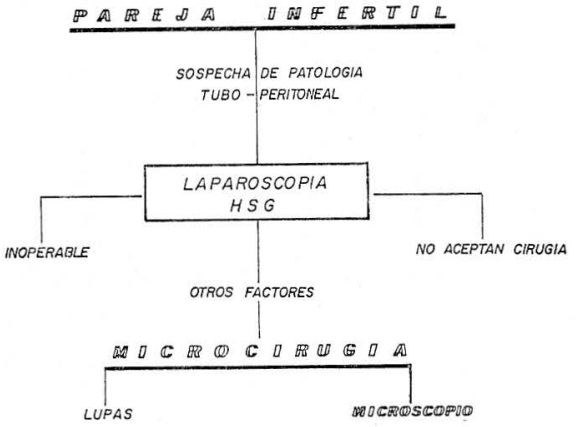

Fig. No. 1 Selección de pacientes para microcirugía tubárica.

Las microcirugías se llevaron a cabo después de la menstruación y en las 2 primeras semanas del ciclo menstrual y siguiendo los principios básicos de la técnica microquirúrgica (fig 3) (15). En todas se empleó el microscopio quirúrgico con magnificaciones entre 10 y 40 . La hemostasia meticulosa se efectuó con microcauterios mono y/o bipolar, se suturó con Nylon monofilamento 8-0 y 9.0 o con apoliglicólico 8-0 y 9-0, los tejidos se movilizaron atratimáticamente $y$ con barras de vidrio y se lavaban continuamente con solución de Ringer mezclada con Heparina e Hidrocortisona en proporción de 1 litro, 5.000 UI y 100 mg. La cromotubación al iniciar el acto quirúrgico y al finalizarlo para establecer la permeabilidad tubárica se realizó trans-

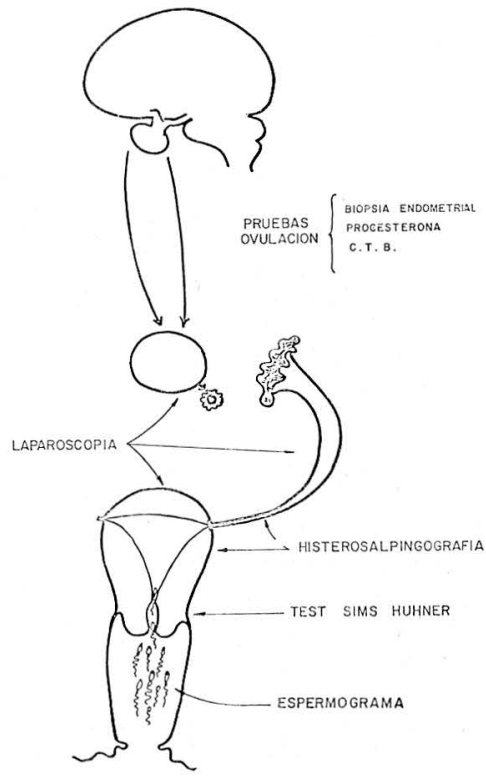

Fig. No. 2 Pruebas básicas en el estudio de la infertilidad.

\section{TECNICA MICROQUIRURGICA}

\section{PRINCIPIOS}

\section{ATRAUMATICA}

2. USO DE MAGNIFICACION

3. HEMOSTASIS METICULOSA

4. PRECISA APROXIMACION DE PLANOS TISULARES

5. USO DE SUTURAS FINAS E INERTES

Fig. No. 3 Principios básicos de la microcirugía.

fúndicamente con azul de metileno y con la ayuda de la pinza de Shirodkar.

Todos los procedimientos se agruparon siguiendo la clasificación internacio- 
nal vigente (fig 4) (16) y durante las microcirugías se efectuaron las variaciones técnicas concernientes a cada grupo. La Salpingo-ovariolisis se realizó utilizando el microelectrodo monopolar e injertos de peritoneo libre cuando fueron necesarios según Wiston (17) y Suchet (18).

\section{ABNORMALITIES RELATED TO INFERTILITY}

1. Ovariolysis

2. Salpingolysis

3. Lysis of adnexal adhesions

4. Tubo uterine implantation

5. Tubo tubo anastomosis

6. Salpingoneostomies

7. Fimbrioplasty

8. Other reconstructive tubal operations

9. Combination of different types of operations

Cognat M Int J Fertil 27:27, 1982

Figura No. 4 Classification of operations for Tuboperitoneal.

Los implantes tubo-uterinos siguiendo la técnica que utiliza un cateter de Nylon 2-0 para retirarlo en el post-operatorio $(17,19,20)$.

Las anastomosis tubo-tubáricas según las técnicas de Gomel $(15,21)$, suturando en 2 planos, músculo-muscular y seroseroso.

Las salpingoneostom ías y fimbrioplastias, resecando el extremo patológico tubárico, haciendo incisión del extremo distal del hidrosalpinx, seccionando la serosa, desaglutinando la fimbria $y$ haciendo eversión en pétalos de rosa $(17$, $22,23,24)$.

100 c.c. Dextran se dejaron intraperitonealmente al concluír la cirugía en algunos casos de lisis de adherencias severas al igual que la utilización de dexametasona post-operatoria.
Las microcirugías se practicaron en un lapso comprendido entre noviembre 1 de 1980 y enero 1 de 1983. El seguimiento ha variado entre 6 y 32 meses en el momento del análisis estadístico, pero está previsto por 3 años para considerar a paciente como éxito o fracaso e incluye una Laparoscopia de control un año después de la microcirugía (fig 5).

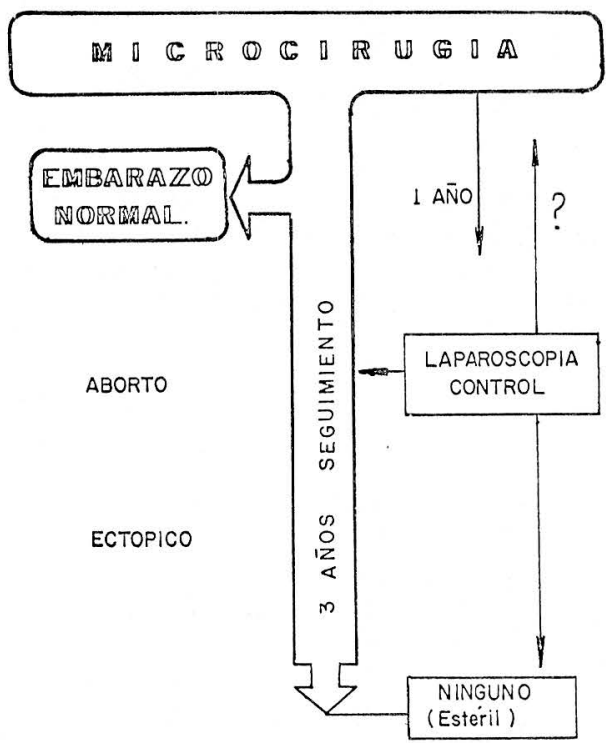

Fig. No. 5 Seguimiento y control post-microcirugía

\section{RESULTADOS}

Salpingo-ovariolisis: 13 de 18 pacientes se embarazaron, con una tasa del $72.2 \%$; 10 pacientes $(55.5 \%)$ han cursado un embarazo normal y 3 han abortado $(16.6 \%)$ (tabla 1$)$.

Implantes tubo-uterinos: 5 de 8 pacientes operadas han quedado embarazadas para una tasa del $62 \% ; 3$ normales $(37.5 \%), 1$ aborto $(12.5 \%) 1$ ectópico $(12.5 \%)$ (tabla 2$)$.

Anastomosis tubo-tubáricas: 6 de 11 han logrado el embarazo, para una tasa 
Tabla No. 1

ADHESIOLISIS

( Ovariolisis - Salpingolisis)

\begin{tabular}{|l|c|c|c|c|}
\hline \multirow{2}{*}{ CAUSAS } & \multirow{2}{*}{$N^{\circ}$ CASOS } & \multicolumn{4}{|c|}{ EMBARAZOS } & $13(72.2 \%$ \\
\cline { 3 - 5 } & & NORMALES & AEORTOS & ECTOPICOS \\
\hline POR ENDOMETRIOSIS & 5 & 4 & - & - \\
POST-INFECCION & 13 & 6 & 3 & - \\
\hline TOTAL & 18 & $10(55.5 \%)$ & $3(16.6 \%)$ & - \\
\hline
\end{tabular}

del $54.5 \%$; 5 han cursado embarazo normal, $(45.5 \%)$ y 1 aborto $(9 \%$ ) (tabla 3 ). Las Anastomosis tubo-tubáricas constituyen el grupo de recanalizaciones post-
Tabla No. 2

IMPLANTES TUBO-UTERINOS

\begin{tabular}{|l|c|c|c|c|}
\hline \multirow{2}{*}{$\begin{array}{c}\text { TIPO OE } \\
\text { MICROCIRUGIA }\end{array}$} & \multirow{2}{*}{ N CASOS } & \multicolumn{3}{|c|}{ EMBARAZOS 5} \\
\cline { 3 - 5 } & & NORMALES & ABORTOS & ECTOPICOS \\
\hline ISTMICO-CORNUAL & 6 & 2 & 1 & - \\
AMPULO-CORNUAL & - & - & - & - \\
COMSINACION & 2 & 1 & - & 1 \\
\hline TOTAL & 8 & $3(37.5 \%)$ & $I(12.5 \%)$ & $1(12.5 \%)$ \\
\hline
\end{tabular}

ligadura de trompas $\mathrm{y}$ en la tabla 3 se presentan los diferentes tipos de anastomosis y sus resultados, también relacionados con la técnica que se había empleado en la ligadura.

Tabla No. 3

ANASTOMOSIS TUBO-TUBARICAS

\begin{tabular}{|c|c|c|c|c|}
\hline \multirow{2}{*}{$\begin{array}{c}\text { TIPO DE } \\
\text { ANASTOMOSIS }\end{array}$} & \multirow{2}{*}{$\begin{array}{l}\text { NO DE } \\
\text { CASOS }\end{array}$} & \multicolumn{3}{|c|}{ EMBARAZOS $6(54.5 \%)$} \\
\hline & & NORMAL & ABORTO & ECTOPICO \\
\hline ISTMICO-ISTMICA & 1 & 1 & & \\
\hline AMPULO- ISTMICA & 7 & 4 & 1 & \\
\hline AMPULO-AMPULAR & 1 & & & \\
\hline COMBINACION & 2 & & & \\
\hline TOTAL & II & $5(45.5 \%)$ & $1(9 \%)$ & \\
\hline
\end{tabular}

\begin{tabular}{|l|l|l|l|l|}
\hline BANDAS YOON & 2 & $2(100 \%)$ & \\
POMEROY & 3 & $1(33.3 \%)$ & $1(33.3 \%)$ & \\
CAUTERIZACION & 2 & $1(50 \%)$ & & \\
POR INFECCION & 4 & $1(25 \%)$ & & \\
\hline
\end{tabular}


Salpingoneostom ías: Solo en 1 de 8 se ha obtenido éxito, para una tasa del $12.5 \%$. El embarazo transcurrió normalmente: (tabla 4).

Tabla No. 4

\section{SALPINGONEOSTOMIAS}

\begin{tabular}{|c|c|c|c|}
\hline \multirow{2}{*}{$N^{\circ}$ CASOS } & \multicolumn{3}{|c|}{ EMBARAZOS $1(12.5 \%)$} \\
\cline { 2 - 4 } & NORMALES & ABORTOS & ECTOPICOS \\
\hline \multirow{2}{*}{8} & $1(12.5 \%)$ & - & - \\
\hline
\end{tabular}

Fimbrioplastias: éxito en 3 de 6 para una tasa del $50 \%$. Dos normales (33. $3 \%$ ) y un aborto $(16.6 \%$ ) (tabla 5$)$.

Combinaciones de procedimientos: 19 pacientes fueron intervenidas combinando adhesiolosis con otros procedimientos tales como fimbrioplastias, salpingoneostomías, anastomosis, miomectomías, e injertos de peritoneo libre; 3 de 19 se embarazaron, para una tasa del $15.9 \%$. Una abortó $(5.3 \%)$ y una terminó en ectópico $(5.3 \%)$ (tabla 6 ).

Los resultados globales se pueden ver
Tabla No. 5

FIMBRIOPLASTIAS

\begin{tabular}{|c|c|c|c|c|}
\hline $\begin{array}{c}\text { TIPO OE } \\
\text { MICROCIRUGIA }\end{array}$ & No CASOS & \multicolumn{3}{|c|}{ EMBARAZOS 3 $(50.0 \%)$} \\
\cline { 3 - 5 } & NORMALES & ABORTOS & ECTOPICOS \\
\hline $\begin{array}{l}\text { OEAGLUTINACION } \\
\text { FIMBRIAS }\end{array}$ & 4 & 1 & 1 & \\
$\begin{array}{l}\text { INCISION OE LA } \\
\text { SEROSA POR } \\
\text { OCLUSION COMFLETA }\end{array}$ & 2 & 1 & & \\
\hline TOTAL & 6 & $2(33.3 \%)$ & $(116.6 \%)$ & \\
\hline
\end{tabular}

Tabla No. 6

\section{COMBINACION DE DIFERENTES TIPOS DE MICROCIRUGIAS}

\begin{tabular}{|c|c|c|c|}
\hline \multirow{2}{*}{$N=$ CASOS } & \multicolumn{3}{|c|}{ № EMBARAZOS $3 \quad(15.9 \%)$} \\
\hline & NORMALES & ABORTOS & ECTOPICOS \\
\hline 19 & $1(5.3 \%)$ & / (5.3\%) & $1(5.3 \%)$ \\
\hline
\end{tabular}

en la tabla 7 en donde además se presentan 2 pacientes a quienes se practicaron cuñas ováricas con técnica microquirúrgica. Estas pacientes que fallaron en la

Tabla No. 7

MICROCIRUGIA GINECOLOGICA

\begin{tabular}{|c|c|c|c|c|c|c|c|c|c|c|c|}
\hline $\begin{array}{c}\text { TIPO DE } \\
\text { MICROCIRUGIA }\end{array}$ & NI CASOS & \multicolumn{2}{|c|}{ NE EMBARAZOS } & \multicolumn{2}{|c|}{$\begin{array}{l}\text { EMBARAZOS } \\
\text { NORMALES }\end{array}$} & \multicolumn{3}{|c|}{ ABORTOS } & \multicolumn{3}{|c|}{ ECTOPICOS } \\
\hline Adhesillosis & 18 & $13-$ & $72.2 \%$ & $10-$ & $55.5 \%$ & 3 & -1 & $16.6 \%$ & 一 & - & - \\
\hline Implantes Tubo_Uterino & 8 & $5-$ & $62.0 \%$ & $3-$ & $37.5 \%$ & 1 & - & $12.5 \%$ & 1 & -1 & $12.5 \%$ \\
\hline Anastomosis Tubo Jubáricos & 11 & $6-$ & $54.5 \%$ & $5-$ & $45.5 \%$ & 1 & - & $9.0 \%$ & - & - & 一 \\
\hline Solpingonoostomias & 8 & $1-$ & $12.5 \%$ & $1-$ & $12.5 \%$ & - & - & - & - & - & - \\
\hline Fimbrioplastios & 6 & $3-$ & $50.0 \%$ & $2-$ & $33.3 \%$ & 1 & - & $16.6 \%$ & - & - & - \\
\hline Combinaciones & 19 & $3-$ & $15.8 \%$ & $1-$ & $5.3 \%$ & 1 & - & $5.3 \%$ & 1 & - & $5.3 \%$ \\
\hline Cuñas Ováricas & 2 & & $100 \%$ & $2-$ & $100 \%$ & - & - & - & - & - & - \\
\hline
\end{tabular}


inducción de la ovulación con Clomifén, se embarazaron post-microcirugía.

\section{COMENTARIOS Y CONCLUSIONES}

La demanda de microcirugía en el tratamiento del factor tubo-peritoneal de infertilidad y para revertir la esterilización quirúrgica ha sido creciente. La enfermedad pélvica inflamatoria, la cirugía pélvica previa y la endometriosis son los factores etiologicos más comunes de la infertilidad tubo-peritoneal, pero también en nuestro medio ha ido aumentando la demanda de reversión de la ligadura de trompas.

La selección de las pacientes para microcirugía debe ser metódica; dentro de un protocolo de manejo de la infertilidad, determinar que los riesgos no sean superiores a los beneficios del procedimiento y ofrecer previa al acto quirúrgico las posibilidades de éxito para cada caso.

El seguimiento debe ser por lo menos de 3 años, ya que las mejores posibilidades de éxito van desde el ciclo siguiente a la microcirugía y hasta 3 años después (14), con un promedio en la ocurrencia del embarazo a los 10.2 meses del postoperatorio (15).

Los éxitos dependen de muchos factores tal como el tipo de patología tubo-peritoneal a operar, edad de la paciente, patología asociada, experiencia del cirujano y disponibilidad de equipos óptimos. Como éxitos definitivos solo se deben considerar los embarazos a término con recién nacidos vivos y como esto depende del tipo de patología peritoneal que presenten las pacientes, los casos y sus resultados deben estar sometidos a una clasificación de procedimientos quirúrgicos para normalidades tubo-peritoneales de infertilidad (16). Esta clasificación además hace posible el poder comparar con mejor aproximación los resultados de diferentes microcirujanos.
Los éxitos obtenidos en el presente trabajo muestran una correlación inversa al proceso patológico tubo-peritoneal que causó la infertilidad. Los mejores grupos son los de las salpingo-ovariolisis y las recanalizaciones post-ligadura y los peores los de las combinaciones de corrección de varios estados patológicos subyacentes $y$ las salpingoneostom ías por hidrosalpinx.

Las futuras pacientes de estos dos últimos grupos tendrán mejores resultados con la fertilización in vitro y transferencia de embriones, pero las de los grupos restantes aún tienen en la microcirugía sus mejores esperanzas de lograr un embarazo normal.

\section{RESUMEN}

El papel de la microcirugía en la recuperación de la fertilidad se presenta en 72 casos de infertilidad por factor tuboperitoneal, operados por el autor en el Hospital Militar y La Clínica San Pedro Claver de Bogotá entre nov. 1 de 1982 y enero 1 de 1983. Las pacientes se seleccionaron siguiendo un estudio básico de la pareja infértil con laparoscopia, histerosalpinqografía, prueba post-coito, espermograma, biopsia endometrial y progesterona mesoluteínica. Las candidatas a microcirugía se clasificaron por grupos patológicos, siguiendo las normas internacionales tanto para la técnica microquirúrgica como para el análisis de los resultados. Las tasas de éxito de embarazos a término o en progresión normal hasta la fecha son las siguientes: salpingo -ovariolisis 55.5\% (10 de 18). Anastomosis tubotubáricas post-ligadura $45.5 \%$ (5 de 11). Implantes tubo-uterinos 37. $5 \%$ (3 de 8). Fimbrioplastias $33.3 \%$ (2 de 6). Salpingoneostomías $12.5 \%$ (1 de 8). Combinación de procedimientos 5.3\% (1 de 19). La microcirugía tubárica reconstructiva continúa siendo el procedimiento terapéutico más importante del factor tubo-peritoneal. 


\section{TREATMENT OF TUBAL PERITONEAL FACTOR THROUGH MICROSURGERY}

\section{SUMMARY}

This article shows the use of microsurgery in the recovery of fertility in 72 cases of infertility due to the tubal peritoneal factor, as performed by the author at Hospital Militar Central and Clínica San Pedro Claver of Bogotá between November 1, 1982 and January $1,1983$.

The patients were selected following basic analyses of the infertile couple through laparoscopy, hysterosalpingography, post-coital test, espermogram, endometrial biopsy and mesolutheinic progesterone. The candidates for microsurgery were separated by groups of pathology, following international criteria for both

\section{BIBLIOGRAFIA}

1. GOMEL V. An odyssey through the oviduct. Fertil Steril 39:144, 1983.

2. PATERSON P, WOOD $C$. The use of microsurgery in the reanastomosis of the rabbit fallopian tube. Fertil Steril 25:757, 1974.

3. WINSTON RML. Microsurgical reanastomosis of the rabbit oviduct and its functional and pathologic sequelae. $\mathrm{Br} \mathrm{J}$ Obstet Gynaecol 82:513, 1975.

4. McCOMB P, GOMEL $\vee$. The influence of fallopian tube lenght on fertility in the rabbit. Fertil Steril 31:673, 1979.

5. MCCOMB P, BOER-MEISEL M, GOMEL $V$. The influence of fallopian tube ampullary lenght on the fertility of the rabbit. Int $\mathrm{J}$ Fertil 26:30, 1981.

6. KHOO SK, MACKAY EV. Reactions in rabbit fallopian tube after plastic reconstruction. I. Gross pathology, tubal patency, and pregnancy. Fertil Steril 23:201, 1972.

7. EDDY CA, ANTONINI R Jr. PAUERSTEIN CJ. Fertility following microsurgical removal the microsurgical technique and analysis of the results.

Success rates of full term pregnancies or pregnancies in normal progression to date are as follows: Salpingo-ovariolisis: $55.5 \%$ (10 out of 18 ). Post-ligation tubal-tubarian anastomosis: $45.5 \% \quad(5$ out of 11). Tubal-uterine implants: 37 . $5 \%$ (3 out of 8 ). Fimbrioplastias: 33.3 (2 out of 6 ). Salpino-neostomies: $12.5 \%$ (1 out of 8 ). Combination of procedures: $5.3 \%$ (1 out of 19 ).

Reconstructive tubarian microsurgery is still the most adequate therapeutic approach in the presence of tubal peritoneal factor.

of the ampullary-isthmic junction in rabbits. Fertil Steril 28:1090, 1977.

8. EDDY CA, HOFFMAN JJ, PAUERSTEIN CJ. Pregnancy following segmental isthmic reversal of the rabbit oviduct. Experientia 32:1194, 1976.

9. PEREZ LE, FLORES JJ, BAJPAI VK, ASCH $\mathrm{RH}$ and EDDY CA. Fertility Following Fimbriectomy and Tubo-Ovarian Microsurgery in the rabbit. Fertil Steril 35:573, 1981.

10. PEREZ LE, RAJKUMAR K, EDDY CA. Fertility and Ovum Transport after Microsurgical Removal of the Uterotubal junction in Rabbits. Fertil Steril 36:803, 1981.

11. PEREZ LE, EDDY CA. Ovum transport and fertility following microsurgical removal of the isthmus and uterotubal junction (UTJ) in rabbits. Biol Reprod 22:72A, 1980.

12. Reversing fernale Sterilization. Population Reports. Series CN.8, 1980.

13. Microsurgery in Female Infertility, edited by P.G. Crosignani and B.L. Rubin; published by Academic Press Inc. (London) Ltda., 24/28 Oval Road, London NWI 7DX, Uk, 
and Gruen and Stratton, 111 fifth Avenue, New York, New York 10003, USA. 1980.

14. UMEZAKI C, KATAYAMA P, JONES $H$. Pregnancy Rates after Reconstructive Surgery on the Fallopian Tubes. Obst Gynecol 43:418, 1974.

15. GOMEL V. Microsurgical Reversal of Female Sterilization: A Reappaisal. Fertil Steril 33: 587, 1980.

16. ROLAND M. A new classification for surgical procedures for tubo-peritoneal abnormalities in infertility.

17. WINSTON RML. Microsurgical Tuboplasty. Hammersmith Hospital, London. Copyright Downs Surgical, 1978.

18. SUCHET, JH, LOFFREDO V. Tratamiento quirúrgico de las esterilidades tubáricas. Interés del injerto de peritoneo libre y de la laparoscopia precoz en la proyección de las adherencias. Presse Médicale 2:341, 1980.
19. WISTON RML. Microsurgical Tubocornual Anastomosis for reversal of sterilization. Lancet 1:284-285, 1977.

20. McCOMB P, GOMEL V. Oclusión Cornual y su reconstrucción microquirúrgica. Clínicas Obstétricas y Ginecológicas 4:1291, 1980.

21. WINSTON RML. Inversión de la esterilización tubárica. Clínicas Obstétricas y Ginecológicas 4:1328, 1980.

22. GOMEL V, SWOLIN K. Salpingostomia: Técnica microquirúrgica y resultados. Clínicas Obstétricas y Ginecológicas 4:1305, 1980.

23. NOVY MJ. Reversal of Kroner fimbriectomy Sterilization J Obstet Gynecol 137:198, 1980.

24. COHEN BM. Surgical Repair of Abnormal Fimbrial Gonadal Relationships in Human Female. J of Reprod med 25:33, 1980. 